\title{
Mathematical Modelling of Pulsatile Flow of Non-Newtonian Fluid Through a Constricted Artery
}

\author{
Saktipada Nanda ${ }^{1}$, Biswadip Basu Mallik ${ }^{2 *}$, Samarpan Deb Majumder ${ }^{3}$, Ramesh Kumar Karthick ${ }^{3}$, Sagar Suman $^{3}$, \\ Sahil Sonkar ${ }^{3}$ \\ ${ }^{1}$ Department of Electronics and Communication Engineering, Institute of Engineering \& Management, Kolkata 700091, India \\ ${ }^{2}$ Department of Basic Science and Humanities, Institute of Engineering \& Management, Kolkata 700091, India \\ ${ }^{3}$ Department of Mechanical Engineering, Institute of Engineering \& Management, Kolkata 700091, India
}

Corresponding Author Email: biswadip.basumallik@iemcal.com

https://doi.org/10.18280/mmep.080320

Received: 24 March 2021

Accepted: 6 May 2021

\section{Keywords:}

CVD (cardiovascular disease), power law fluid model, pulsatile flow, slip condition, stenosis

\begin{abstract}
The research work explores blood flow into a stenosed artery, or one with abnormal growth within it. At the throats and at the critical height of the stenosis, mathematical and computational models have been developed to calculate the various associated parameters such as flow rate, pressure gradient, impedance, and wall shear stress. Modeling blood as a power law fluid showed the dependency of these quantities on temporal and spatial variables, as well as the frequency of the flow oscillation in time and the key parameters of the flow mechanism. The exponential curve is the geometry of the stenosis studied in this analysis. Analytical expressions for axial velocity, volumetric flow rate, pressure gradient, blood flow resistance, and shear stress have been computed and simulated in ANSYS to generate useful results with respect to variation of flow parameters with power law indices and also for comparison between Newtonian and Non- Newtonian models of blood. Upon investigation, it was found that wall shear stress (WSS) increases with stenosis depth and therefore, plays a crucial role in affecting other flow parameters. At power law index 0.6, the highest shear stress and flow velocity were encountered at approximately $7 \mathrm{~Pa}$ and $0.5 \mathrm{~m} / \mathrm{s}$ respectively.
\end{abstract}

\section{INTRODUCTION}

One of the main objectives outlined in the United Nations 2030 action plan for sustainable development is to reduce onethird of the premature deaths from non-communicable diseases [1]. The world's most common non-communicable diseases are CVDs (Cardio-vascular diseases), with more than $50 \%$ of such deaths occurring in low- and intermediate-income countries in 2017, with 17.8 million deaths in 2017 [2]. According to data from 79 countries (mostly low and middleincome countries), in 79 countries (mostly low- and mediumincome nations), in a recent report published by the World Health Organisation, the figures for people aged 40-64 who are more than $20 \%$ at risk of cardiovascular disease are estimated as being higher than $20 \%$. (Mainly countries with low and medium revenues) [3].

The actual causes of the formation of the undesirable growth in the arterial lumen is yet to be investigated from the stand point of physiology/pathology. It is estimated that the mechanical distinctive speciality of the conducting tube for blood flow together with the discontinuity (or, irregularity) of blood streaming through the vessels and unusual deposit in the inner side of the artery are considered as the cause and development of many critical hematological problems.

The hematological disease 'Carotid Circulatory Disorders' is due to constriction of blood flow in the artery, the constriction is developed for unusual growths inside. It plays a significant role in the occurrence of many severe cardiovascular diseases like ischemia, stroke and heart attack.
The soft target of the disease are the older people with blood sugar, blood pressure etc. The exact reason behind the occurrence of the fatal disease is ambiguous and the opinion of the investigators vary to some extent. The rheological and physical nature of the blood together with the geometry of the stenosis might significantly contribute to the genesis and development of the hematological diseases. If the problem is neglected at the initial stage of attack and allowed to proceed to an advanced level then the outcome might be insufficient flow of blood to brain, heart and other organs or rupture of the artery due to high concentration of pressure. It would be high risk for survival leading to morbidity or even death [4-6].

The temporary oxygen deficiency or improper supply of blood to the heart is the main cause of heart related diseases. This deficiency is owing to the hindrance in sufficient blood supply due to constrictions developed in the blood vessels or arteries. Constriction is caused by the accumulation of fatty substances such as cholesterol, cellular waste products, calcium, and other substances in the inner lining of the artery. The unusual growth due to the deposition is called stenosis. They restrict the normal movement of blood where the flow of blood to the region beyond the narrowing reduces considerably $[7,8]$. The stenosis disrupts the natural flow of blood, resulting in the development of atherosclerosis and a heart attack.

A wide variety of analytical as well as experimental studies on streaming of blood through the branches of the arterial chain having a single or multiple stenoses were carried out by several investigators applying different blood models 
(Newtonian or non-Newtonian) and various geometry of the stenosis (polynomial, smooth cosine or exponential). Young [9] presented the effects of arterial stenosis on non-Newtonian blood flow in an artery, while Shukla et al. [10] studied the effects of stenosis on non-Newtonian blood flow in an artery. Some theories suggest that irregular growth in an artery lumen can be initiated by cholesterol deposition on the arterial wall and connective tissue growth. While the exact causes of stenosis are still unclear, the effects can be seen in blood flow in the area on the cardiovascular system. The presumption of Newtonian blood activity has been indicated to be appropriate for high shear rate flow by Tu et al. [11]. Tu and Deville [12] have also examined pulsative blood flow in stenosed arteries. In presence of applied magnetic field effects with velocity and temperature fields, the Newtonian blood flow model was provided by Kumar and Diwakar [13]. Via elastic artery, the analysis of blood flow using power legislation and HerschelBulkley (non-Newton fluid models) were discussed by Kumar [14]. Chitra and Karthikeyan [15] created a mathematical model of a non-Newtonian pulse fluid flow through an elastic artery with elasticity effects. Similar studies were conducted to study the blood flow under various other influencing external conditions such as sudden shocks, magnetic field exposure, and many more [16-18]

The rheological activity of blood in small diameter tubes (less than $0.2 \mathrm{~mm}$ ) at shear rate $20 \mathrm{sec}^{-1}$ can be fairly interpreted by the Power Law Fluid Model, according to Whitmore [19]. The arterial tube is under pressurized condition and therefore will possess slipping action (a velocity discontinuity) between the fluid and arterial wall. So, the slip condition is given due consideration in the present study. The geometry of the stenosis affects the blood flow. There are many types of stenoses like cosine shaped stenosis, bellshaped stenosis, overlapping stenosis, irregular stenosis, multi- irregular stenosis etc. Based on the research and literature study [20], the geometry of stenosis taken here is that of a bell-shape.

A rigorous study of the previous literature on hematological problems due to uncommon growth within the artery and consequently irregularity in blood transmission or sometimes total obstruction led to construct the fluid model for blood, standard geometrical model for the growth together with the boundary conditions.

The analytical expressions for various blood flow parameters have been derived. An intellectually stimulating model of human narrow artery/vein constricted due to unnatural growths covered in the analysis of hematological problems are presented. On comparing the analytical results with the software simulation results, conclusions were drawn The results may be useful in the medical and pharmaceutical industries and could improve the precision and quality of treatment given to people suffering from the deadly disease.

\section{METHODOLOGY}

As shown in Figure 1, the cardiovascular fluid-blood (modeled as a power law fluid) is flowing through a circular cross-section artery with stenosis at a particular location. It is assumed that the flow is laminar, viscous, and incompressible. The artery's length is believed to be sufficient in comparison to its diameter, allowing the end results to be ignored. It is due to the fact that the pressure is relatively high in the vicinity of the growth not at the ends. So, the contribution of pressure at the ends may be neglected. A mathematical model of the stenosis in the artery was developed, assuming a power law fluid model for blood and bell shape geometry of the stenosis. In the boundary conditions, slip (discontinuity in the wall) condition is imposed.

The study and analysis done is a comparative one. The comparison has been done between the pulsatile nature and steady nature of blood flow. Blood flow velocity, volumetric flow rate, pressure drop across the stenosed area, flow impedance, and wall shear stress were all measured at first, and analytical expressions were established. The analysis of the stenosed artery has been done by virtue of computational fluid dynamics (CFD) in ANSYS, where the results for the variation of the flow parameters like, blood flow velocity, wall shear stress etc. with respect to power law indices and position of stenosis, were generated for both pulsatile flow as well as steady flow.

Firstly, the mathematical formulation of the problem along with requisite assumptions and notations would be defined. This would be followed by presentation of the analysis of the problem. The results of the analytical equations would be worked out. This would succeed the CFD Simulation of the problem in ANSYS and finally validation of the solution would be done for the extent of agreement of the simulation results with the numerical investigation. The conclusion and inferences would be laid down from validation of results.

\subsection{Mathematical formulation}

The Geometry of Stenosis manifested in the artery is given below:

$$
\frac{R(z)}{R_{0}}=\left\{\begin{array}{l}
1-\frac{\delta}{R_{0}} \cdot \exp \left[\frac{w^{2} \varepsilon^{2} z^{2}}{R_{0}^{2}}\right] \\
1 ; \quad \text { otherwise }
\end{array}\right\}
$$

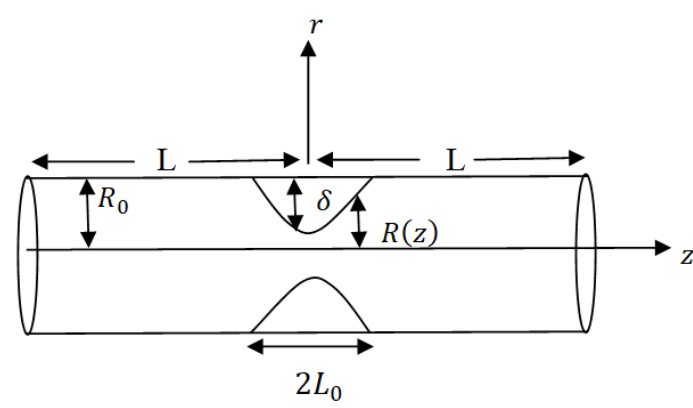

Figure 1. Geometry of stenosed artery (Bell shaped)

$\mathrm{R}$ refers to the non-stenotic radius of the arterial segment. $\mathrm{R}(\mathrm{z})$ is the radius of the axial distance $\mathrm{z}$ of the section on the left end of the artery.

$\delta$ is the stenosis's peak point,

$w$ is a parametric constant,

$\varepsilon$ is relative length of the constriction, i.e. $\frac{R_{0}}{L_{0}}$.

The main equations of the fluid model of power law are,

$$
\begin{aligned}
\tau & =\mu e^{n} \\
\tau & =\frac{1}{2} p r
\end{aligned}
$$




$$
e=\frac{-d u}{d r}
$$

The boundary conditions are:

$u=u_{s}$ at $r=\mathrm{R}(\mathrm{z})$ [velocity slip condition]. $\tau$ is finite at $r=0$ [Regularity condition].

$\frac{d u}{d r}=0$ at $r=0$ [Velocity gradient is 0 ].

Combining Eqns. (2), (3) \& (4), we have,

$$
\frac{\partial u}{\partial r}=-\left(\frac{1}{2} \frac{p}{\mu}\right)^{\frac{1}{n}} r^{\frac{1}{n}}
$$

Integrating for $u$,

$$
\begin{aligned}
& \int d u=-\left(\frac{1}{2} \frac{P}{u}\right)^{\frac{1}{n}} \int r^{\frac{1}{n}} d r \\
& u=-\left(\frac{1}{2} \frac{p}{\mu}\right)^{\frac{1}{n}} r^{\frac{n+1}{n}} \frac{n}{n+1}+C \\
& u=-\left(\frac{1}{2} \frac{p}{\mu}\right)^{\frac{1}{n}} r^{\frac{n+1}{n}} \frac{n}{n+1}+C
\end{aligned}
$$

Putting boundary conditions as $u=u_{s}$ at $r=\mathrm{R}(z)$ :

$$
\begin{gathered}
u_{s}=-\left(\frac{1}{2} \frac{p}{\mu}\right)^{\frac{1}{n}} \frac{R^{\frac{n+1}{n}} n}{n+1}+C \\
C=\left(\frac{1}{2} \frac{p}{\mu}\right)^{\frac{1}{n}} \frac{R^{\frac{n+1}{n}} n}{n+1}+u_{s} \\
u=-\left(\frac{1}{2} \frac{p}{\mu}\right)^{\frac{1}{n}} \frac{r^{\frac{n+1}{n}} n}{n+1}+\left(\frac{1}{2} \frac{p}{\mu}\right)^{\frac{1}{n}} \frac{R^{\frac{n+1}{n}} n}{n+1}+u_{s} \\
u=\frac{n}{n+1}\left(\frac{1}{2} \frac{P}{\mu}\right)^{\frac{1}{n}}\left[R^{\frac{n+1}{n}}-r^{\frac{n+1}{n}}\right]+u_{s}
\end{gathered}
$$

Now, calculate the volumetric flow rate,

$$
\begin{gathered}
Q=\int_{0}^{R} 2 \pi u r d r \\
Q=\int_{0}^{R} 2 \pi\left[\frac{n}{n+1}\left(\frac{1}{2} \frac{P}{\mu}\right)^{\frac{1}{n}}\left(R^{\frac{n+1}{n}}-r^{\frac{n+1}{n}}\right)+u_{s}\right] r d r \\
Q=\frac{n}{n+1}\left(\frac{1}{2} \frac{P}{\mu}\right)^{\frac{1}{n}} \pi R^{\frac{2 n+1}{n}}\left(1-\frac{2 n}{3 n+1}\right)+\pi u_{s} R^{2} \\
Q=\frac{n}{3 n+1} \pi R^{\frac{3 n+1}{n}}\left(\frac{1}{2} \frac{P}{\mu}\right)^{\frac{1}{n}}+\pi u_{s} R^{2}
\end{gathered}
$$

Pressure Gradient $P$ is given as $\frac{d p}{d z}$. From Eq. (7),

$$
P=\frac{d p}{d z}=2 \mu\left[\left(Q-\pi u_{s} R^{2}\right)\left(\frac{3 n+1}{n \pi R^{\frac{3 n+1}{n}}}\right)\right]^{n}
$$

Integrating for Pressure drop, we get,

$$
\begin{gathered}
\int_{-p}^{p} d p=2 \mu\left(3+\frac{1}{n}\right)^{n} \int_{-l}^{l}\left[\left(\frac{Q}{\pi R^{\frac{3 n+1}{n}}}-\frac{u_{s}}{R^{\frac{n+1}{n}}}\right)\right]^{n} d z \\
\int_{0}^{p} d p=2 \mu\left(3+\frac{1}{n}\right)^{n} \int_{0}^{l}\left[\left(\frac{Q}{\pi R^{\frac{3 n+1}{n}}}-\frac{u_{s}}{R^{\frac{n+1}{n}}}\right)\right]^{n} d z \\
\Delta p=2 \mu\left(3+\frac{1}{n}\right)^{n} \int_{0}^{l_{0}}\left[\left(\frac{Q}{\pi R^{\frac{3 n+1}{n}}}-\frac{u_{s}}{R^{\frac{n+1}{n}}}\right)\right]^{n} d z+ \\
\int_{l_{0}}^{l-l_{0}}\left[\left(\frac{Q}{\pi R_{0} \frac{3 n+1}{n}}-\frac{u_{s}}{R_{0}^{\frac{n+1}{n}}}\right)\right]^{n} d z
\end{gathered}
$$

$$
\begin{gathered}
\Delta p=2 \mu\left(3+\frac{1}{n}\right)^{n}\left\{\left(\frac{Q}{\pi R} \frac{u_{s} \frac{3 n+1}{n}}{R^{\frac{n+1}{n}}}\right)\right]^{n} l_{0}+\left[\left(\frac{Q}{\pi R_{0}^{\frac{3 n+1}{n}}}-\right.\right. \\
\left.\left.\left.\frac{u_{S}}{R_{0} \frac{n+1}{n}}\right)\right]^{n}\left(l-l_{0}\right)\right\}
\end{gathered}
$$

Pressure difference without stenosis, i.e. at $\delta=0$ is obtained as,

$$
\Delta p_{(\delta=0)}=2 \mu\left(3+\frac{1}{n}\right)^{n}\left[\left(\frac{Q}{\pi R_{0} \frac{3 n+1}{n}}-\frac{u_{s}}{R_{0} \frac{n+1}{n}}\right)\right]^{n} l
$$

Thus,

$$
\frac{\Delta p}{\Delta p_{(\delta=0)}}=\frac{\left.\left.\left\{\left[\left(\frac{Q}{\pi R}-\frac{u_{S}}{n}\right)\right]_{R}^{n}\right]_{0}^{\frac{n+1}{n}}\right)\left[\left(\frac{Q}{\pi R_{0} \frac{3 n+1}{n}}-\frac{u_{S}}{R_{0} \frac{n+1}{n}}\right)\right]^{n}\left(l-l_{0}\right)\right\}}{\left[\left(\frac{Q}{\pi R_{0} \frac{3 n+1}{n}}-\frac{u_{S}}{R_{0} \frac{n+1}{n}}\right)\right]^{n} l}
$$

Expression for impedance or flow resistance $\tilde{\lambda}$ is given as,

$$
\begin{aligned}
\tilde{\lambda}=\lambda_{0}= & \frac{\Delta p}{Q}=\frac{2 \mu}{Q}\left(3+\frac{1}{n}\right)^{n}\left\{\left[\left(\frac{Q}{\pi R^{\frac{3 n+1}{n}}}-\frac{u_{s}}{R^{\frac{n+1}{n}}}\right)\right]^{n} l_{0}+\right. \\
& {\left.\left[\left(\frac{Q}{\pi R_{0} \frac{3 n+1}{n}}-\frac{u_{s}}{R_{0} \frac{n+1}{n}}\right)\right]^{n}\left(l-l_{0}\right)\right\} }
\end{aligned}
$$

Impedance without stenosis, i.e. at $\delta=0$ is obtained as,

$$
\lambda_{N}=\frac{2 \mu}{Q}\left[\left(\frac{3 n+1}{n}\right)\left(\frac{Q}{\pi R_{0} \frac{3 n+1}{n}}-\frac{u_{S}}{R_{0}^{\frac{n+1}{n}}}\right)\right]^{n} l
$$

Therefore, the non-dimensional flow resistance form is obtained as:

$$
\frac{\lambda_{0}}{\lambda_{N}}=\frac{\left\{\left[\left(\frac{Q}{\pi R^{\frac{3 n+1}{n}}}-\frac{u_{S}}{R^{\frac{n+1}{n}}}\right)\right]^{n} l_{0}+\left[\left(\frac{Q}{\pi R_{0} \frac{3 n+1}{n}}-\frac{u_{S}}{R_{0} \frac{n+1}{n}}\right)\right]^{n}\left(l-l_{0}\right)\right\}}{\left[\left(\frac{Q}{\pi R_{0} \frac{3 n+1}{n}}-\frac{u_{S}}{R_{0} \frac{n+1}{n}}\right)\right]^{n} l}
$$

In the stenotic field, the wall shear stress is given by,

$$
\begin{gathered}
\tau_{w}=\frac{-R}{z} \frac{d p}{d z}=\frac{-R}{z} 2 \mu\left[\left(Q-\pi u_{s} R^{2}\right)\left(\frac{3 n+1}{n \pi R^{\frac{3 n+1}{n}}}\right)\right]^{n} \\
\tau_{N}=\frac{-R_{0}}{z} 2 \mu\left[\left(Q-\pi u_{S} R_{0}^{2}\right)\left(\frac{3 n+1}{n \pi R_{0} \frac{3 n+1}{n}}\right)\right]^{n}
\end{gathered}
$$

The non- dimensional form of wall shear stress at stenosis throat is given by,

$$
\tau=\frac{\tau_{w}}{\tau_{N}}=\frac{\left[\left(Q-\pi u_{S} \mathrm{R}^{2}\right)\right]^{n}}{\left[\left(Q-\pi u_{S} R_{0}^{2}\right)\right]^{n}}\left[\frac{R_{0}}{\mathrm{R}}\right]^{3 n}
$$

\subsection{Experimental Setup \& Practical Implementation}

In this section, the experimental setup in ANSYS has been demonstrated and the related computational formulations. 
2.2.1 Development of experimental model in ANSYS for CFD analysis

The geometry of stenosed artery depicted in Figure 2 were generated based on the Eq. (1)

Table 1 lists the different input parameters used in the analysis.

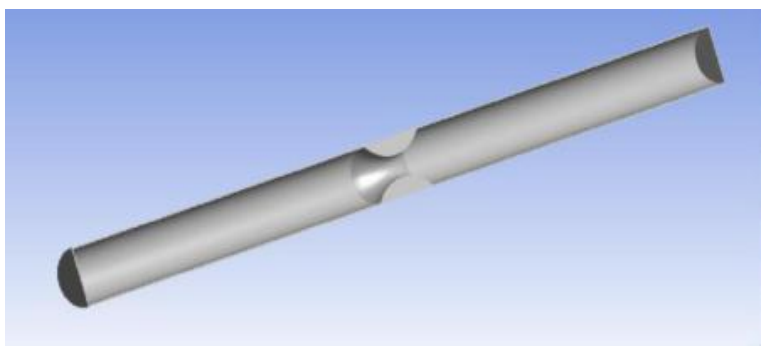

Figure 2. Isometric View of Stenosed Artery

Table 1. Input parameters

\begin{tabular}{cc}
\hline Parameters & Magnitude \\
\hline *Mid RCA Radius & $0.1565 \mathrm{~cm}$ \\
$\frac{\delta}{R_{0}}$ & 0.2 \\
$* * \mathrm{k}$ & 0.017 \\
$\mathrm{n}_{1}$ & 0.33 \\
$\mathrm{n}_{2}$ & 0.66 \\
$\mathrm{n}_{3}$ & 1.00
\end{tabular}

**Mid RCA Radius has been taken as per Normal Coronary Artery Dimensions in Indians [5].

${ }^{*} \mathrm{k}$ is consistency index.

The value of consistency index in the power law equation has been considered to be 0.017 as proposed by Ali and Sen [6] The geometry of artery under analysis was discretized into large number of elements, the details of which are mentioned in Table 2. To minimize computational error and time, the grid independency test was used to calculate the mesh density.

Table 2. Parameters in mesh analysis in ANSYS

\begin{tabular}{cccc}
\hline$\frac{\boldsymbol{\delta}}{\boldsymbol{R}_{\mathbf{0}}}$ & 0.6 & 0.4 & 0.2 \\
\hline Number of Nodes & 10375 & 10292 & 9052 \\
Number of Elements & 9052 & 9102 & 7872 \\
Tetrahedra & 0 & 0 & 0 \\
Wedges & 248 & 492 & 492 \\
Pyramids & 0 & 0 & 0 \\
Hexahedra & 8804 & 8610 & 7380 \\
Polyhedra & 0 & 0 & 0 \\
\hline
\end{tabular}

\subsection{CFD Simulation using ANSYS}

The non-Newtonian behavior of blood flow cannot be ignored in a variety of geometry, according to the literature on blood rheology [7, 8]. Blood is a thinning, shear fluid, which increases the shear rate and becomes less viscous. Blood was supposed to be a fluid for the CFD analysis that is a nonNewtonian fluid, or a generalized Newtonian one, which calculates shear stress given by:

$$
\tau=k\left[\frac{d u}{d y}\right]^{n}
$$

where,

$\mathrm{K}$ is the flow consistency index (SI unit $\left.\mathrm{Pa}-\mathrm{s}^{\mathrm{n}}\right)$, $\frac{d u}{d y}$ is the shear rate or velocity gradient perpendicular to the plane of shear (SI unit s ${ }^{-1}$ ),

$n$ is the flow behaviour index (dimensionless).

The quantity, $\mu_{e_{f f}}=k\left[\frac{d u}{d y}\right]^{n-1}$ represents an apparent or effective viscosity as a function of the shear rate (SI unit Pas).

The CFD analysis has been done for two cases of blood flow, first for pulsatile flow and second assuming a mean velocity of the blood, i.e. steady flow.

By enforcing a variable speed limit at the entrance, pulsatile flow was used in the simulation. The pulsational profile is considered to be a two-phase combination within each cycle. The speed of the inlet differs in sinusoidal design during the systolic process. In the systolic phase, the sine wave has a top speed of $0.5 \mathrm{~m} / \mathrm{s}$ and a minimum speed of $0.1 \mathrm{~m} / \mathrm{s}$. It takes 0.5 seconds each time and takes 120 beats a minute at a heart rate. The interruption in heart supply is represented by a constant speed, i.e. a steady diastole flow. For the proposed inlet velocity model, a user defined function (udf) was written. This model of pulsatile flow, as given in Figure 4, is taken as proposed by Sinnot et al. [4].

A steady flow limit of $0.315 \mathrm{~m} / \mathrm{s}$ was used to compare the steady flow and pulsatile flow (See Figure 3).

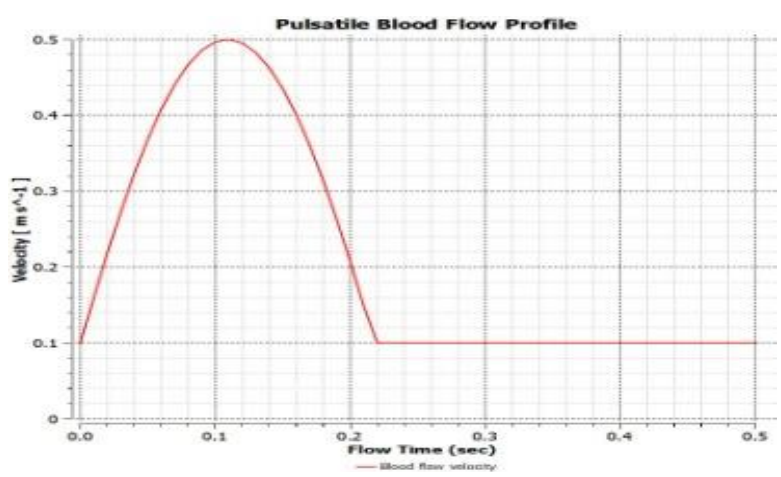

Figure 3. Pulsatile velocity profile

\section{RESULTS AND DISCUSSIONS}

Due to the pulsating character of the flow, simulations were run for 10 time steps of $0.05 \mathrm{~s}$ to analyze the difference in velocity distribution and artery wall shear stresses. This time interval, as shown in Figure 4, corresponds to a pulsative flow period, with a systolic phase of up to $0.218 \mathrm{~s}$ and a diastolic phase of $0.218 \mathrm{~s}$ to $0.5 \mathrm{~s}$.

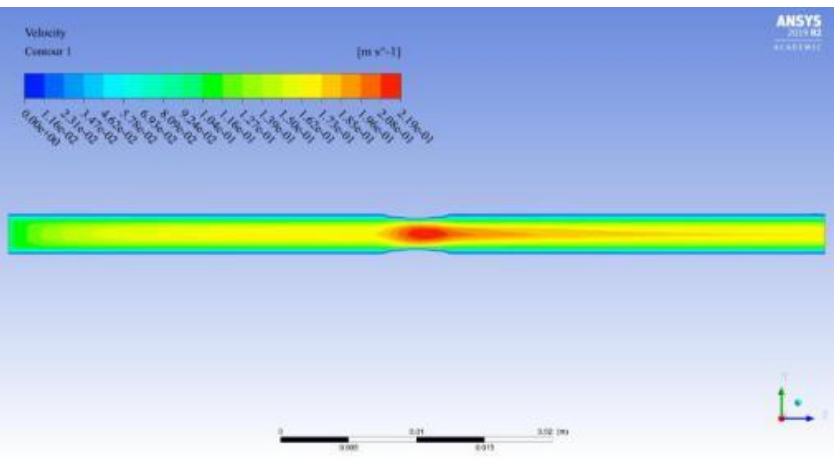

Figure 4. Velocity Distribution for $\frac{\delta}{R_{0}}=0.2$ 


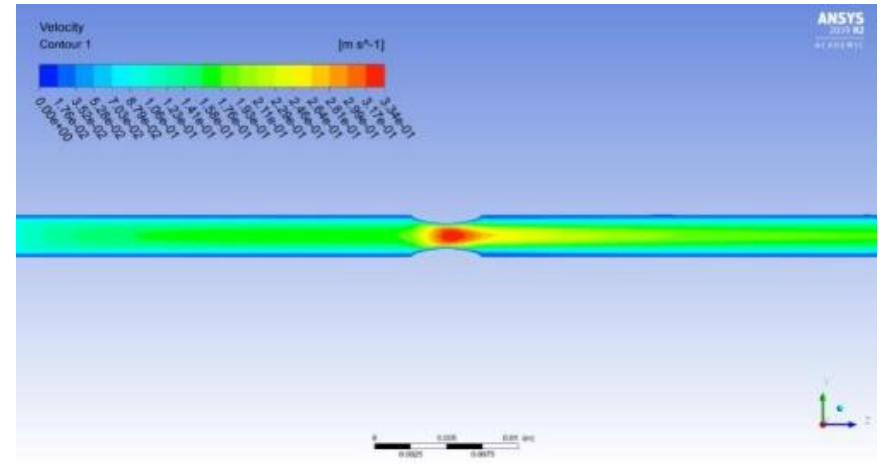

Figure 5. Velocity Distribution for $\frac{\delta}{R_{0}}=0.4$

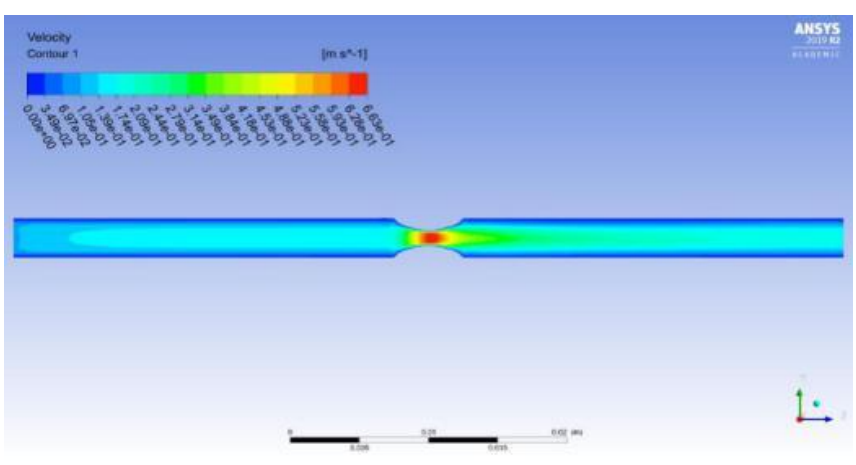

Figure 6. Velocity Distribution for $\frac{\delta}{R_{0}}=0.6$

Figure 4, Figure $5 \&$ Figure 6 illustrate the contours of velocity through the stenosed artery and Figure 7, Figure $8 \&$ Figure 9 demonstrates the contours of WSS through the stenosed artery for different values of $\frac{\delta}{R_{0}}$.

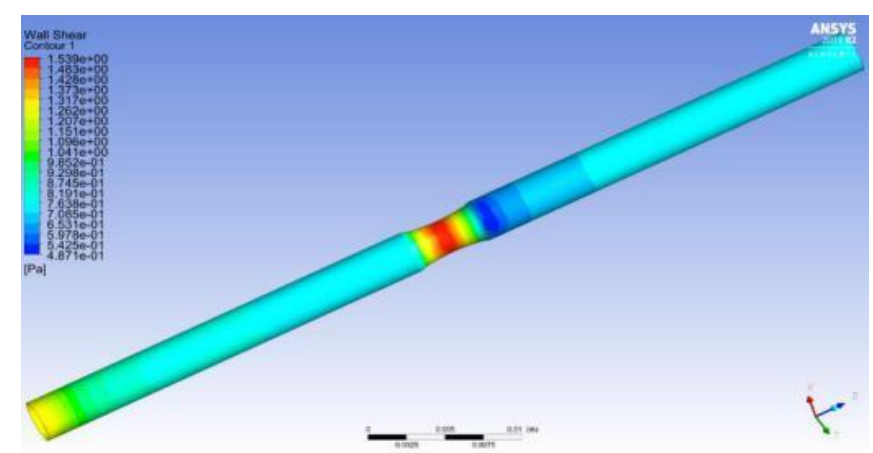

Figure 7. WSS Distribution for $\frac{\delta}{R_{0}}=0.2$

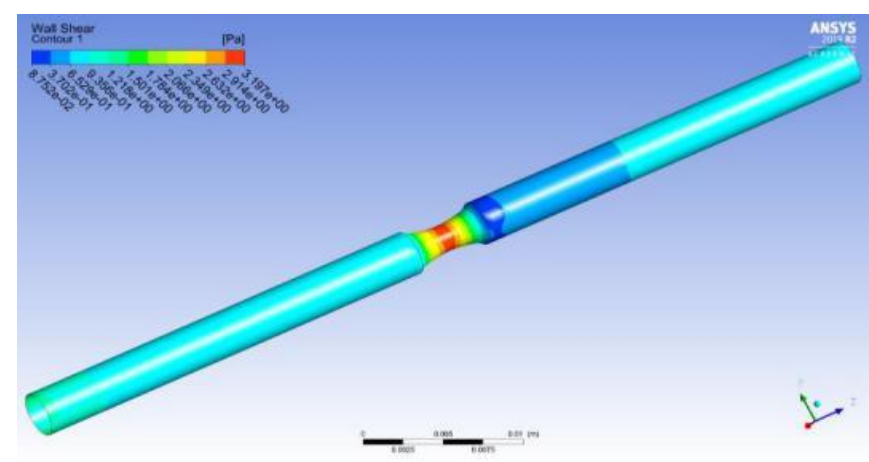

Figure 8. WSS Distribution for $\frac{\delta}{R_{0}}=0.4$

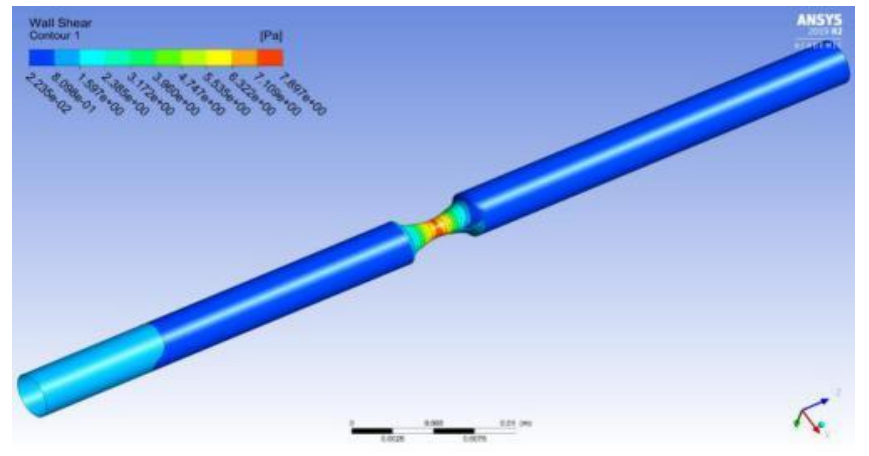

Figure 9. WSS Distribution for $\frac{\delta}{R_{0}}=0.6$

Figure 10 and Figure 11 represent variation of Velocity and WSS with power law index (n) respectively, for both steady and pulsatile flow of blood through the stenosed artery. From the graphs it is clear that with increase in power law index (n), there is an increase in velocity \& WSS. It can be clearly inferred that the values of WSS (wall shear stress) and velocity obtained for steady state condition is nearly double the values obtained for pulsatile blood flow condition.

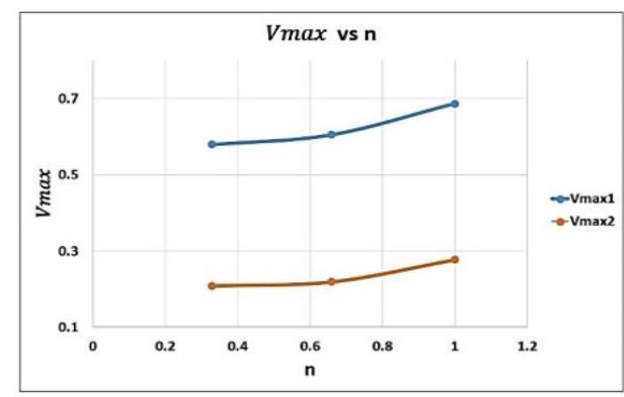

Figure 10. Velocity profile comparison $\left(\mathrm{V}_{\max 1-S t e a d y}\right.$ flow; $\mathrm{V}_{\max 2-}$ Pulsatile Flow)

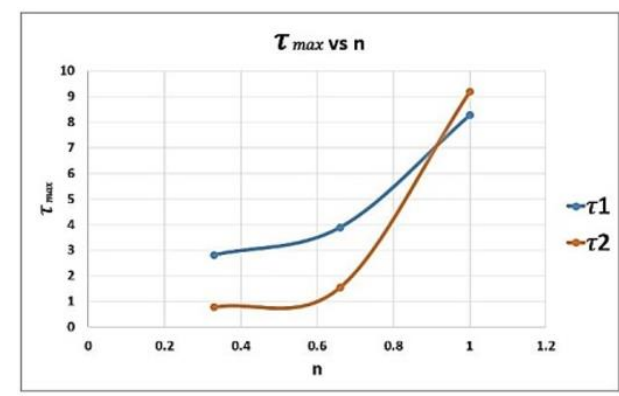

Figure 11. Wall shear stress comparison ( $\tau_{1}$ - Steady flow; $\tau_{2}$ Pulsatile Flow)

Figure 12 exhibits the variation of WSS along the artery for different values of $\frac{\delta}{R_{0}}$ (i.e. $0.6,0.4 \& 0.2$ ) for a given power law index value $(\mathrm{n}=0.67)$, with stenosis- symmetrical about the center. Clearly, for a given value of $n$, the WSS increases with increasing $\frac{\delta}{R_{0}}$ values and is significantly higher at higher $\frac{\delta}{R_{0}}$ values.

Figure 13 and Figure 14 show the time variation of velocity and WSS. Table 4 shows the time step data for the variation of velocity and WSS in the artery (for the second cycle). Table 3 shows that maximum values of flow parameters (in the second cycle) initially increase with time until $\mathrm{t}=0.6 \mathrm{~s}$, then begin to 
decrease until $\mathrm{t}=0.8 \mathrm{~s}$, and then remain nearly constant thereafter. This is so, since there is sinus (systole phase) blood flow before $\mathrm{t}=0.218 \mathrm{~s}$, followed by $0.5 \mathrm{~m} / \mathrm{s}$ peaks at $\mathrm{t}=0.1 \mathrm{~s}$. In the first stage of the diastolic process $(\mathrm{t}=0.218$ to $0.5 \mathrm{~s})$, the rate of inlet is constant $(0.1 \mathrm{~m} / \mathrm{s})$. This means that the supply to heart is reduced, resulting in blood flow constantly and hence the values of flow parameters become constant.

The relation of blood flow as a power law fluid with a power law index of $n=1$ (Newtonian) and $n=0.67$ (Non-Newtonian) are shown in Figure 15 and Figure 16. From Figure 15 it can be observed that the velocity profile is nearly identical in both the cases whereas, Figure 16 illustrates that the WSS in case of Newtonian fluid is almost triple that of Non-Newtonian case. The time step data for $n=1$ is given in Table 4 .

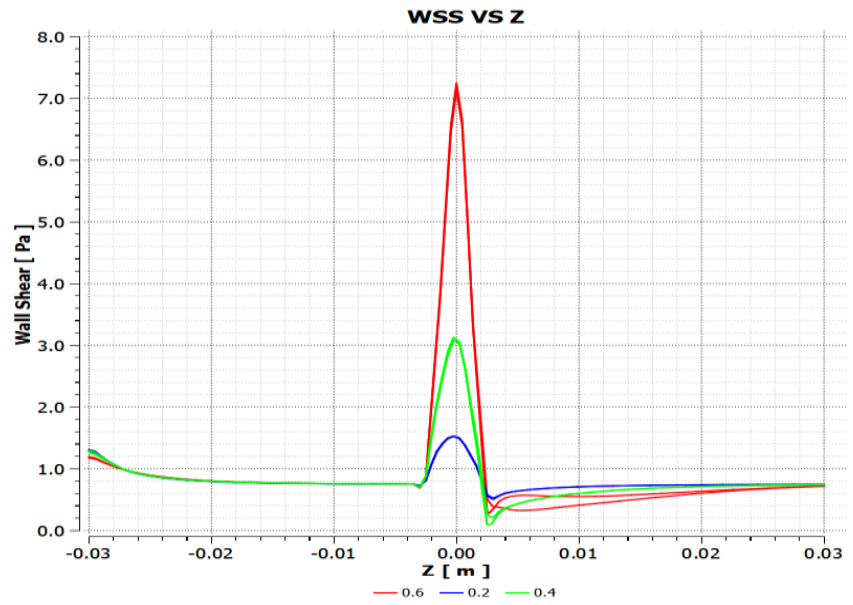

Figure 12. Variation of WSS along the length of the artery

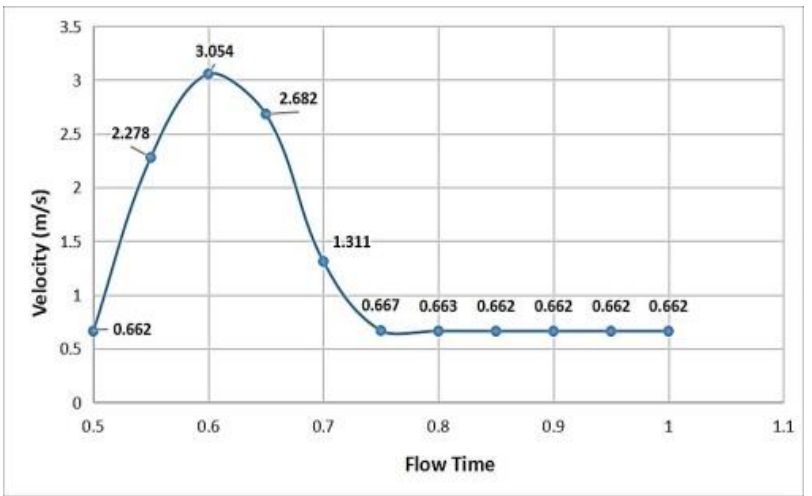

Figure 13. Velocities at different time intervals

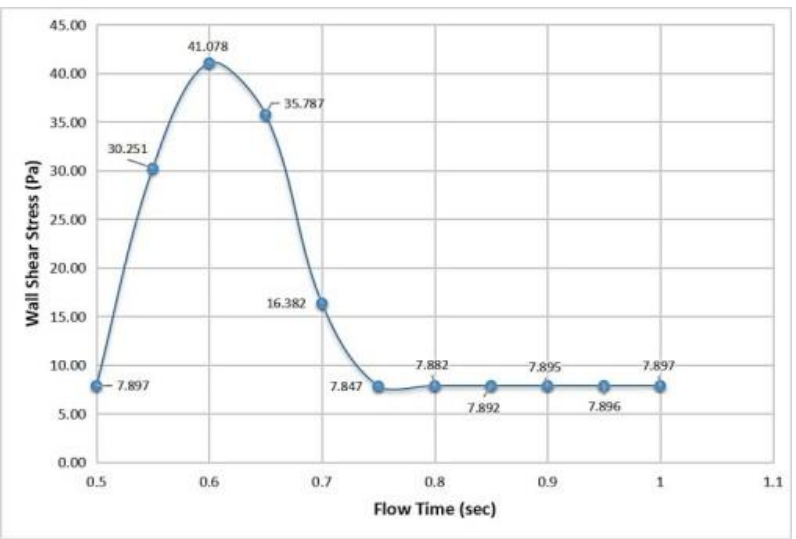

Figure 14. WSS at different time intervals
Table 3. Flow Parameters at different time intervals for $\mathrm{n}=0.67$ (Non-Newtonian)

\begin{tabular}{ccc}
\hline Time (s) & Maximum Velocity (m/s) & Maximum WSS (Pa) \\
\hline 0.50 & 0.662 & 7.897 \\
0.55 & 2.278 & 30.251 \\
0.60 & 3.054 & 41.078 \\
0.65 & 2.682 & 35.787 \\
0.70 & 1.311 & 16.382 \\
0.75 & 0.667 & 7.847 \\
0.80 & 0.663 & 7.882 \\
0.85 & 0.662 & 7.892 \\
0.90 & 0.662 & 7.895 \\
0.95 & 0.662 & 7.896 \\
1.00 & 0.662 & 7.897 \\
\hline
\end{tabular}

Table 4. Flow Parameters at different time intervals for $n=1$ (Newtonian)

\begin{tabular}{ccc}
\hline Time (s) & Maximum Velocity (m/s) & Maximum WSS (Pa) \\
0.55 & 2.394 & 84.644 \\
0.60 & 3.178 & 134.54 \\
0.65 & 2.808 & 116.294 \\
0.70 & 1.444 & 50.409 \\
0.75 & 0.772 & 21.934 \\
1.00 & 0.767 & 22.113 \\
\hline
\end{tabular}

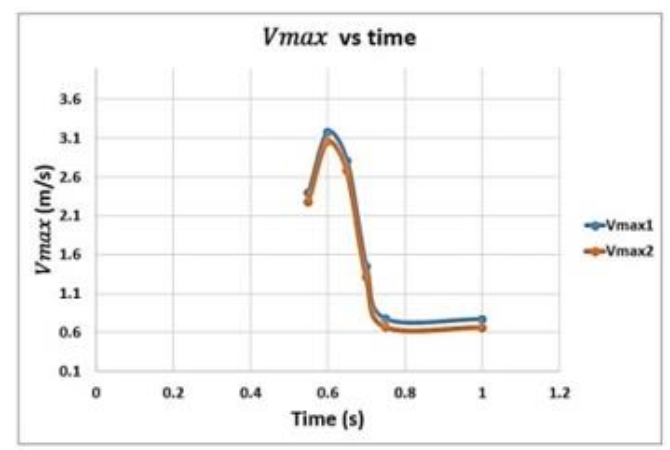

Figure 15. Velocity profile comparison $\left(\mathrm{V}_{\max 1}-\mathrm{Newtonian}\right.$; $\mathrm{V}_{\max 2}$ - Non-Newtonian)

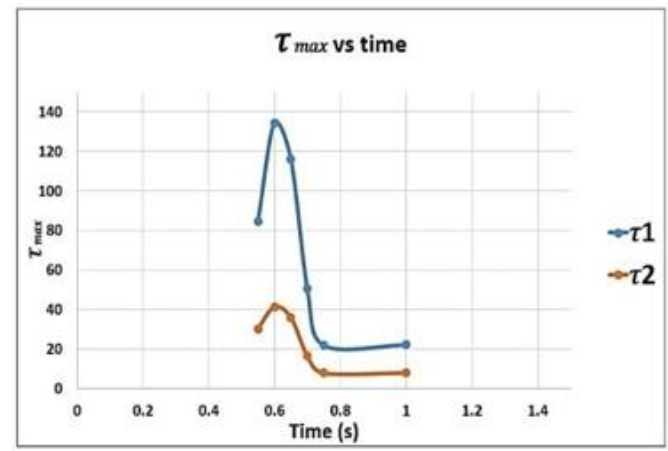

Figure 16. Wall shear stress comparison

\section{CONCLUSIONS}

This study presents a mathematical modeling and CFD study of blood flow in a stenosed coronary artery, with blood assumed to be a power law fluid. Comparison of the flow parameters, i.e. speed and wall shear stress for various power law index values (n) were presented. Experiments were also performed to compare the variation of velocity and WSS with time assuming blood as a Newtonian fluid $(n=1)$ and Non- 
Newtonian fluid $(n=0.67)$. The velocity and wall shear stress magnitudes increased with power law index because of the presence of arterial stenosis. The variance of wall shear stress (WSS) for different depths of stenosis was also investigated for a given value of $n$. The WSS was found to increase significantly as the depth of stenosis increased. The presence of a high WSS is considered an important factor in stenosis development and progression. These factors cause blood pressure to increase, downstream flow to decrease, and the percentage of stenosis to rise. In the pulsatile case, the transient flow field varies significantly from the steady flow field. By approximately doubling the flow parameter values, the steady flow field verifies them (velocity and WSS). To study the influence of flow parameters on stenosis progression, more studies that are closer to the physical scenario are needed. It is not claimed that this investigation and the output will replace the modern techniques of medical and surgical treatments of the diseases but they may add some additional inputs in their detection and initial treatment.

\section{REFERENCES}

[1] United Nations. Transforming our world: the 2030 agenda for sustainable development. https://www.un.org/ga/search/view_doc.asp?symbol=A/ RES/70/1\&Lang=E.

[2] Roth, G.A., Abate, D., Abate, K.H. et al. (2018). Global, regional, and national age-sex-specific mortality for 282 causes of death in 195 countries and territories, 1980 2017: A systematic analysis for the Global Burden of Disease Study 2017. The Lancet, 392(10159): 17361788. https://doi.org/10.1016/S0140-6736(18)32203-7

[3] Lancet Glob Health 2019 Published Online September 2, 2019. http://dx.doi.org/10.1016/S2214-109X(19)303183

[4] Sinnott, M., Cleary, P.W., Prakash, M. (2006). An investigation of pulsatile blood flow in a bifurcation artery using a grid-free method. Fifth International Conference on CFD in the Process Industries, CSIRO, Melbourne, Australia

[5] Saikrishna, C., Talwar, S., Gulati, G., Kumar, A.S (2006). Normal coronary artery dimensions in Indians. Indian Journal of Thoracic and Cardiovascular Surgery, 22(3): 159-164. https://doi.org/10.1007/s12055-0060750-2

[6] Ali, D., Sen, S. (2018). Permeability and fluid flowinduced wall shear stress of bone tissue scaffolds: computational fluid dynamic analysis using Newtonian and non-Newtonian blood flow models. Computers in Biology and Medicine, 99: 201-208. https://doi.org/10.1016/j.compbiomed.2018.06.017

[7] Gijsen, F.J., van de Vosse, F.N., Janssen, J.D. (1999). The influence of the non-Newtonian properties of blood on the flow in large arteries: Steady flow in a carotid bifurcation model. Journal of Biomechanics, 32(6): 601608. https://doi.org/10.1016/S0021-9290(99)00015-9

[8] Moore, S., David, T., Chase, J.G., Arnold, J., Fink, J. (2006). 3D models of blood flow in the cerebral vasculature. Journal of Biomechanics, 39(8): 1454-1463. https://doi.org/10.1016/j.jbiomech.2005.04.005

[9] Young, D.F. (1979). Fluid mechanics of arterial stenoses. J Biomech Eng, 101(3): 157-175 https://doi.org/10.1115/1.3426241
[10] Shukla, J.B., Parihar, R.S., Rao, B.R.P. (1980). Effects of stenosis on non-Newtonian flow of the blood in an artery. Bulletin of Mathematical Biology, 42(3): 283-294. https://doi.org/10.1007/BF02460787

[11] Tu, C., Deville, M., Dheur, L., Vanderschuren, L. (1992). Finite element simulation of pulsatile flow through arterial stenosis. Journal of Biomechanics, 25(10): 1141 1152. https://doi.org/10.1016/0021-9290(92)90070-H

[12] Tu, C., Deville, M. (1996). Pulsatile flow of nonNewtonian fluids through arterial stenoses. Journal of Biomechanics, 29(7): 899-908. https://doi.org/10.1016/0021-9290(95)00151-4

[13] Kumar, S., Diwakar, C.S. (2012). A mathematical model for Newtonian blood flow in the presence of applied magnetic field. Aryabhatta J. of Math \& Infomatics, 4: 265-278.

[14] Kumar, S. (2015). Study of blood flow using Power law and Harschel-Bulkley non-Newtonian fluid model through elastic artery. Proceedings of ICFM, pp. 229-235.

[15] Chitra, M., Karthikeyan, D. (2017). Mathematical modelling of pulsatile flow of non-Newtonian fluid through an elastic artery: Effects of elasticity. International Journal of Pure and Applied Mathematical Sciences, 10(1): 29-40.

[16] Nanda, S., Basu Mallik, B., Singh, C., Das, S., Ghosh, S., Bhattacharya, S., Chatterjee, S.S. (2017). Study on the effect of non Newtonian nature of blood flowing through an elastic artery in slip condition. Journal of Chemical, Biological and Physical Sciences, 7(4): 934-942. https://doi.org/10.24214/jcbps.C.7.4.93442

[17] Astrita, G., Marucci, G. (1974). Principles of NonNewtonian Fluid Mechanics, McGraw Hill, New York, USA.

[18] McDonald, D.A. (1974). Blood flow in arteries, Edward Arnold Publishers, London.

[19] Whitmore, R.L. (1968). Rheology of the Circulation, Perg. New York.

[20] Mazumder, J.N. (1992). Biofluid Mechanics, World Scientific Publishing Co. Singapore.

\section{NOMENCLATURE}

$\mathrm{R}_{0} \quad$ the arterial segment's radius in the non-stenotic area

$\mathrm{R}(\mathrm{z})$ radius of artery with stenosis at the axial distance $\mathrm{z}$ from the left end of segment

$\delta \quad$ height of the stenosis

$w$ parametric constant

$\varepsilon \quad$ relative length of the constriction

$\zeta \quad$ Wall shear stress

$u \quad$ final velocity

$\mu \quad$ apparent viscosity of blood

$P \quad$ pressure gradient

$\chi \quad$ flow resistance

$u_{s} \quad$ slip velocity

$k \quad$ consistency index

RCA right coronary artery

WSS wall shear stress

$\mathrm{Z} \quad$ vector along arterial length

CVD Cardiovascular disease

$n \quad$ Power law index 